\title{
Finite Difference Approximations of Generalized Solutions
}

\author{
By Endre Süli, Boško Jovanović and Lav Ivanović
}

\begin{abstract}
We consider finite difference schemes approximating the Dirichlet problem for the Poisson equation. We provide scales of error estimates in discrete Sobolev-like norms assuming that the generalized solution belongs to a nonnegative order Sobolev space.
\end{abstract}

1. Introduction. Recently, there have been many theoretical advances in constructing finite difference schemes approximating boundary value problems for partial differential equations with generalized solutions belonging to Sobolev spaces. For example, Lazarov [4] presents a finite difference approximation of the Dirichlet problem for the Poisson equation with a generalized solution belonging to the Sobolev space $W^{k, 2}$ of integer order $k=2,3$ using the so-called Bramble-Hilbert lemma [1].

Unfortunately, the Bramble-Hilbert lemma is stated only for integer-order Sobolev spaces. Recently, Dupont and Scott [3] gave a constructive proof of this lemma using an averaged Taylor series and extended it to fractional-order Sobolev spaces.

In this paper a basic framework is given which allows the application of the finite difference method in order to approximate generalized solutions belonging to Sobolev spaces $W^{s, p}, 0 \leqslant s \leqslant 4,1<p<\infty$ (Theorems 1 and 3). Proofs are based on the Dupont-Scott approximation theorem.

We shall prove a discrete interpolation inequality (Lemma 2) which will enable us to derive several scales of error estimates (Theorems 2 and 4).

For simplicity, the analysis in this paper only deals with the Dirichlet problem for the Poisson equation in rectangular domains. Extensions to other elliptic boundary value problems in less special domains or to nonlinear problems are possible.

2. Preliminaries and Notations. Let $\mathscr{A}$ be an open rectangle in two-dimensional Euclidean space $\mathbf{R}^{2}$ and $1<p<\infty$. Throughout the paper $W^{s, p}(\mathscr{A})$ is the Sobolev space of order $s \geqslant 0$ (cf. [8]) equipped with the Sobolev norm

$$
\|u\|_{s, p . \mathscr{A}}^{p}=\sum_{k=0}^{s}|u|_{k, p, \mathscr{A}}^{p}
$$

with

$$
|u|_{k, p, \mathscr{A}}^{p}=\sum_{|\alpha|=k}\left\|D^{\alpha} u\right\|_{L^{p}(\mathscr{A})}^{p}
$$

Received May 16, 1983; revised September 14, 1984.

1980 Mathematics Subject Classification. Primary 65N05, 65N10.

1985 American Mathematical Society $0025-5718 / 85 \$ 1.00+\$ .25$ per page 
if $s$ is integer, and

$$
\|u\|_{s, p, \mathscr{A}}^{p}=\|u\|_{[s], p, \mathscr{A}}^{p}+|u|_{s, p, \mathscr{A}}^{p},
$$

if $s=[s]+\sigma$, with $[s]=$ integral part of $s, 0<\sigma<1$ and

$$
|u|_{s, p, \mathscr{A}}^{p}=\sum_{|\alpha|=[s]} \int_{\mathscr{A}} \int_{\mathscr{A}} \frac{\left|D^{\alpha} u(x)-D^{\alpha} u(y)\right|^{p}}{|x-y|^{2+\alpha p}} d x d y .
$$

$\mathbf{N}$ will stand for the set of nonnegative integers. $\mathbf{P}^{\prime}(\mathscr{A})$ will denote the set of polynomials in two variables of degree $\leqslant l$ over the set $\mathscr{A}$, for any $l \in \mathbf{N}$.

The next lemma is an easy consequence of the Dupont-Scott approximation theorem [3] (the case $\sigma=1, p=2$ follows from the Bramble-Hilbert lemma [1]).

Lemma 1. Suppose $s=l+\sigma$, where $0<\sigma \leqslant 1$ and $l \in \mathbf{N}$. Let $\eta$ be a bounded linear functional on $W^{s, p}(\mathscr{A})$ such that $\mathbf{P}^{\prime}(\mathscr{A}) \subset \operatorname{kernel}(\eta)$. There exists a positive constant $C$ (depending on $\mathscr{A}, s, p)$ such that for any $u \in W^{s, p}(\mathscr{A})$

$$
|\eta(u)| \leqslant C|u|_{s, p, \mathscr{A}}
$$

Remark 1. Lemma 1 also follows from the Tartar lemma [2].

Remark 2. If $\eta(u)=0$ for some polynomials of degree $>l$, then an analogous estimate is valid, containing only a part of the seminorm $|u|_{s, p, \mathscr{A}}$ (cf. Lazarov [4], $s \in N, p=2)$.

Let $\mathscr{D}^{\prime}(\mathcal{O})$ denote the space of distributions on $\mathcal{O}$, for any open set $\mathcal{O} \subset R^{2}$. Define the diffe rential operator $\Delta$ on $\mathscr{D}^{\prime}(\mathcal{O})$ by

$$
\Delta u=\frac{\partial^{2} u}{\partial x_{1}^{2}}+\frac{\partial^{2} u}{\partial x_{2}^{2}} .
$$

Let us assume, for the sake of simplicity, that $\Omega$ is an open rectangle in $R^{2}$ with boundary $\partial \Omega$, and consider the Dirichlet problem

$$
\begin{array}{clrl}
\Delta u & =-f & & \text { in } \Omega, \\
u & =0 & & \text { on } \partial \Omega .
\end{array}
$$

By changing variables, we may suppose, without loss of generality, that the rectangle is

$$
\Omega=(0, \pi) \times(0, \pi) .
$$

Throughout the paper we assume that (1) has a unique generalized (distributional) solution in $W^{s, p}(\Omega), 0 \leqslant s \leqslant 4,1<p<\infty$, satisfying (2) in the sense of trace theorems [5], [8].

3. Mollifiers. Consider the function

$$
S_{\nu}(x)= \begin{cases}\left(\frac{\sin (x / 2)}{x / 2}\right)^{\nu}, & x \neq 0 \\ 1, & x=0\end{cases}
$$

with $\nu \in \mathbf{N}$. By the Paley-Wiener-Schwartz theorem [7] there exists a distribution $\boldsymbol{\theta}_{\nu}$ with compact support and with a Fourier transform equal to $S_{\nu}$. 
Remark 3. An easy argument shows that $\theta_{0}$ is the Dirac distribution. For $\nu \geqslant 1, \theta_{\nu}$ is a regular distribution. For example,

$$
\begin{aligned}
& \theta_{1}(x)= \begin{cases}1, & x \in\left(-\frac{1}{2}, \frac{1}{2}\right), \\
0, & x \notin\left(-\frac{1}{2}, \frac{1}{2}\right),\end{cases} \\
& \theta_{2}(x)= \begin{cases}1-|x|, & x \in(-1,1), \\
0, & x \notin(-1,1),\end{cases} \\
& \theta_{3}(x)= \begin{cases}\frac{1}{2}\left(x+\frac{3}{2}\right)^{2}, & x \in\left(-\frac{3}{2},-\frac{1}{2}\right], \\
\frac{3}{4}-x^{2}, & x \in\left[-\frac{1}{2},+\frac{1}{2}\right], \\
\frac{1}{2}\left(x-\frac{3}{2}\right)^{2}, & x \in\left[+\frac{1}{2},+\frac{3}{2}\right), \\
0, & x \notin\left(-\frac{3}{2},+\frac{3}{2}\right) .\end{cases}
\end{aligned}
$$

Let $\nu=\left(\nu_{1}, \nu_{2}\right), \nu_{1}, \nu_{2} \in \mathbf{N}, x=\left(x_{1}, x_{2}\right) \in \mathbf{R}^{2}, \theta_{\nu}$ the tensor product of distributions $\theta_{\nu_{1}}$ and $\theta_{\nu_{2}}, G_{\nu}$ a distribution defined by

$$
G_{\nu}(x)=\frac{1}{h^{2}} \theta_{\nu}\left(\frac{x}{h}\right), \quad h>0,
$$

and $u \in \mathscr{D}^{\prime}\left(R^{2}\right)$. The operator $T_{\nu}$ given by

$$
T_{\nu} u=u * G_{\nu}
$$

will be called mollifier.

Remark 4. Since $G_{\nu}$ is a distribution with compact support, the convolution $u * G_{\nu}$ is well defined.

For $h>0$ and $\nu=\left(\nu_{1}, \nu_{2}\right)$, we set

$$
\Omega_{\nu}=\left\{x=\left(x_{1}, x_{2}\right) \in \mathbf{R}^{2}: h \nu_{i} / 2<x_{i}<\pi-h \nu_{i} / 2, i=1,2\right\} .
$$

Let $u \in \mathscr{D}^{\prime}(\Omega)$ and $u^{*} \in \mathscr{D}^{\prime}\left(R^{2}\right)$ be any extension of $u$. $T_{\nu} u$ will denote the restriction of $T_{\nu} u^{*}$ to $\Omega_{\nu}$.

Remark 5. Let us observe that $T_{\nu} u$ is well defined since it does not depend on $u^{*}$.

For simplicity, we shall write $T_{\nu_{1} \nu_{2}}$ instead of $T_{\left(\nu_{1}, \nu_{2}\right)}$.

4. Construction of Difference Schemes. Pick a nonnegative integer $N>2$ and let $h=\pi / N$. We define the following grids

$$
\begin{aligned}
\mathbf{R}_{h}^{2} & =\left\{x=\left(x_{1}^{\left(i_{1}\right)}, x_{2}^{\left(i_{2}\right)}\right) \in \mathbf{R}^{2}: x_{j}^{\left(i_{1}\right)}=i_{j} \cdot h,\left|i_{j}\right|<\infty, j=1,2\right\}, \\
\omega_{h} & =\Omega \cap \mathbf{R}_{h}^{2}, \quad \gamma_{h}=\partial \Omega \cap \mathbf{R}_{h}^{2}, \\
\bar{\omega}_{h} & =\omega_{h} \cup \gamma_{h}, \quad \gamma_{h}^{1}=\gamma_{h} \cap(\{0, \pi\} \times(0, \pi)), \\
\gamma_{h}^{2} & =\gamma_{h} \cap((0, \pi) \times\{0, \pi\}), \\
\gamma_{h}^{3} & =\gamma_{h} \cap(\{0\} \times(0, \pi) \cup(0, \pi) \times\{0\}), \\
\omega_{h}^{+} & =\omega_{h} \cup \gamma_{h}^{3} .
\end{aligned}
$$

For $v$, a function of discrete arguments, defined on $\mathbf{R}_{h}^{2}$, set

$$
\begin{array}{ll}
\left(\nabla_{j} v\right)(x)=\frac{v\left(x+e_{j} h\right)-v(x)}{h}, & j=1,2, \\
\left(\bar{\nabla}_{j} v\right)(x)=\frac{v(x)-v\left(x-e_{j} h\right)}{h}, & j=1,2,
\end{array}
$$


with $e_{1}=(1,0), e_{2}=(0,1)$, and define

$$
\Delta_{h} v=\bar{\nabla}_{1} \nabla_{1} v+\bar{\nabla}_{2} \nabla_{2} v
$$

A function $v$ of discrete arguments defined on $\omega_{h}$ (or on $\bar{\omega}_{h}$ and equal to zero on $\left.\gamma_{h}\right)$ is said to belong to $L^{p}\left(\omega_{h}\right), 1<p<\infty$, if there exists a positive constant $M$, such that

$$
\|v\|_{p, h}=\left\{h^{2} \sum_{x \in \omega_{h}}|v(x)|^{p}\right\}^{1 / p} \leqslant M
$$

uniformly in $h$.

Remark 6. If $v$ is defined on $\omega_{h}^{+}$(or on $\bar{\omega}_{h}$ and equal to zero on $\gamma_{h} \backslash \gamma_{h}^{3}$ ) the norm $\|\cdot\|_{p, h}$ is replaced by

$$
\|\left[v \|_{p, h}=\left\{h^{2} \sum_{x \in \omega_{h}^{+}}|v(x)|^{p}\right\}^{1 / p} .\right.
$$

Let us suppose that $v$ is defined on $\omega_{h}$ (or on $\bar{\omega}_{h}$ and equal to zero on $\gamma_{h}$ ). The discrete Fourier transform $\tilde{v}$ of $v$ is given by

$$
\tilde{v}_{k}=\sum_{x \in \omega_{h}} h^{2} v(x) \sin \left(k_{1} \cdot x_{1}\right) \sin \left(k_{2} \cdot x_{2}\right), \quad k=\left(k_{1}, k_{2}\right), x=\left(x_{1}, x_{2}\right) .
$$

The inverse discrete Fourier transform of $v$ is defined by

$$
\hat{v}(x)=\left(\frac{2}{\pi}\right)^{2} \sum_{k \in K_{h}} v_{k} \sin \left(k_{1} \cdot x_{1}\right) \sin \left(k_{2} \cdot x_{2}\right),
$$

with $K_{h}=\left\{k=\left(k_{1}, k_{2}\right) \in \mathbf{N} \times \mathbf{N}: 0<k_{j} h<\pi, j=1,2\right\}$.

A function $v$, defined on $\omega_{h}$ (or on $\bar{\omega}_{h}$ and equal to zero on $\gamma_{h}$ ), is said to belong to $W^{r, p}\left(\omega_{h}\right),-\infty<r<\infty, 1<p<\infty$, if there exists a function $V \in L^{p}\left(\omega_{h}\right)$ such that

$$
v(x)=\left(I_{r, h} V\right)(x)=\overbrace{\left(1+|k|^{2}\right)^{-r / 2} \tilde{V}_{k}} .
$$

By definition, we set

$$
\|v\|_{r, p, h}=\|V\|_{p, h} .
$$

We now turn to a generalization of a discrete interpolation inequality established by Mokin [6].

LEMMA 2. Let $\alpha$ and $\beta$ be two nonnegative real numbers such that $\alpha<\beta$. If $v \in W^{\beta, p}\left(\omega_{h}\right), 1<p<\infty$, there exists a positive constant $C$, independent of $h$, such that for any real number $r, \alpha \leqslant r \leqslant \beta$,

$$
\|v\|_{r, p, h} \leqslant C\|v\|_{\alpha, p, h}^{1-\mu}\|v\|_{\beta, p, h}^{\mu},
$$

with $\mu=(r-\alpha) /(\beta-\alpha)$.

Proof. Since the statement is true for $\alpha=0$ [6], we shall assume that $\alpha>0$. Let $w=I_{-\alpha, h} v$. It follows that

$$
\|w\|_{\beta-\alpha, p, h}=\|v\|_{\beta, p, h} \quad \text { and } \quad\|w\|_{r-\alpha, p, h}=\|v\|_{r, p, h} .
$$


Moreover,

$$
\|w\|_{r-\alpha, p, h} \leqslant C\|w\|_{p, h}^{1-\mu}\|w\|_{\beta-\alpha, p, h}^{\mu}
$$

and the desired inequality follows immediately.

Consider the finite difference scheme

$$
\begin{gathered}
-\Delta_{h} z=\bar{\nabla}_{1} \nabla_{1} \eta_{1}+\bar{\nabla}_{2} \nabla_{2} \eta_{2}, \quad x \in \omega_{h}, \\
z(x)=0, \quad x \in \gamma_{h},
\end{gathered}
$$

with $\eta_{j}$ defined on $\omega_{h} \cup \gamma_{h}^{j}$ and equal to zero on $\gamma_{h}^{j}, j=1,2$.

An easy argument based on the discrete multiplicator techniques [6] shows that

$$
\begin{aligned}
\|z\|_{2, p, h} & \leqslant C\left(\left\|\bar{\nabla}_{1} \nabla_{1} \eta_{1}\right\|_{p, h}+\left\|\bar{\nabla}_{2} \nabla_{2} \eta_{2}\right\|_{p, h}\right), \\
\|z\|_{1, p, h} & \leqslant C\left(\|\left[\nabla_{1} \eta_{1}\left\|_{p, h}+\right\|\left[\nabla_{2} \eta_{2} \|_{p, h}\right),\right.\right. \\
\|z\|_{p, h} & \leqslant C\left(\left\|\eta_{1}\right\|_{p, h}+\left\|\eta_{2}\right\|_{p, h}\right),
\end{aligned}
$$

with a positive constant $C$, independent of $z$ and $h$.

Let us suppose that the solution $u$ of boundary value problem (1), (2) belongs to $W^{s, p}(\Omega), s>2 / p, 1<p<\infty$. By Sobolev's imbedding theorem [8], $u$ is continuous on $\Omega \cup \partial \Omega$ and

$$
\begin{aligned}
& \left(T_{20} \frac{\partial^{2} u}{\partial x_{1}^{2}}\right)(x)=\bar{\nabla}_{1} \nabla_{1} u(x), \quad x \in \omega_{h}, \\
& \left(T_{02} \frac{\partial^{2} u}{\partial x_{2}^{2}}\right)(x)=\bar{\nabla}_{2} \nabla_{2} u(x), \quad x \in \omega_{h} .
\end{aligned}
$$

Therefore,

$$
\begin{gathered}
\bar{\nabla}_{1} \nabla_{1} T_{02} u+\bar{\nabla}_{2} \nabla_{2} T_{20} u=-\left(T_{22} f\right)(x), \quad x \in \omega_{h}, \\
u(x)=0, \quad x \in \gamma_{h} .
\end{gathered}
$$

Thus, if the solution of boundary value problem $(1)$, (2) belongs to $W^{s, p}(\Omega)$, $s>2 / p, 1<p<\infty$, we may associate with (1), (2) the finite difference scheme

$$
\begin{gathered}
\Delta_{h} v=-\left(T_{22} f\right)(x), \quad x \in \omega_{h}, \\
v(x)=0, \quad x \in \gamma_{h} .
\end{gathered}
$$

Error estimates will be given in Section 5 .

Let us turn to the case when $u$, the solution of boundary value problem (1), (2), belongs to $W^{s, p}(\Omega), 0 \leqslant s<1+1 / p, 1<p<\infty$. Define $\dot{W}^{s, p}(\Omega)=\left\{\begin{array}{l}W^{s, p}(\Omega), \quad 0 \leqslant s \leqslant 1 / p, 1<p<\infty, \\ \left\{w: w \in W^{s, p}(\Omega), w=0 \text { on } \partial \Omega\right\}, 1 / p<s<1+1 / p, 1<p<\infty\end{array}\right.$ and observe that $u \in \dot{W}^{s, p}(\Omega), 0 \leqslant s<1+1 / p, 1<p<\infty$. Let $\Omega^{*}=(-\pi, 2 \pi)$ $\times(-\pi, 2 \pi)$. The extension of $u$ by 0 outside $\Omega$ is a continuous mapping of $\dot{W}^{s, p}(\Omega)$ into $W^{s, p}\left(\Omega^{*}\right), 0 \leqslant s<1+1 / p, s \neq 1 / p, 1<p<\infty$ [5], [8]. Hence,

$$
u \mapsto u^{*}=\text { odd extension of } u
$$

is a continuous mapping of $\dot{W}^{s, p}(\Omega)$ into $W^{s, p}\left(\Omega^{*}\right), 0 \leqslant s<1+1 / p, s \neq 1 / p$, $1<p<\infty$. 
It is obvious that

$$
\begin{array}{ll}
\left(T_{11} u^{*}\right)(x)=0, & x \in \gamma_{h}, \\
\left(T_{31} u^{*}\right)(x)=0, & x \in \gamma_{h}^{1}, \\
\left(T_{13} u^{*}\right)(x)=0, & x \in \gamma_{h}^{2} .
\end{array}
$$

We introduce $f^{*} \in \mathscr{D}^{\prime}\left(\Omega^{*}\right)$ by

$$
f^{*}=-\Delta u^{*}
$$

Whence,

$$
\begin{gathered}
\bar{\nabla}_{1} \nabla_{1} T_{13} u^{*}+\bar{\nabla}_{2} \nabla_{2} T_{31} u^{*}=-\left(T_{33} f^{*}\right)(x), \quad x \in \omega_{h}, \\
\left(T_{11} u^{*}\right)(x)=0, \quad x \in \gamma_{h} .
\end{gathered}
$$

Therefore, if the solution of (1), (2) belongs to $W^{s, p}(\Omega), 0 \leqslant s<1+1 / p$, $s \neq 1 / p, 1<p<\infty$, we may associate with (1), (2) the finite difference scheme

$$
\begin{gathered}
\Delta_{h} v=-\left(T_{33} f^{*}\right)(x), \quad x \in \omega_{h}, \\
v(x)=0, \quad x \in \gamma_{h} .
\end{gathered}
$$

Error estimates will be given in Section 5.

\section{Convergence of Finite Difference Schemes.}

THEOREM 1. Let $u$ be the solution of boundary value problem (1), (2), $v$ the solution of the discrete problem (10), (11) and $k \in\{0,1,2\}$. If $u \in W^{s, p}(\Omega), 2 / p<s \leqslant k+2$, $1<p<\infty$, the following error estimate holds

$$
\|u-v\|_{k, p, h} \leqslant C h^{s-k}|u|_{s, p, \Omega},
$$

with a positive constant $C$ independent of $h$. Moreover, if $s \geqslant k$ then finite difference scheme (10), (11) converges in the discrete norm $\|\cdot\|_{k, p, h}$.

Proof. (a) Let us suppose first that $k=2$. By (8)-(11) it follows that the function $z=v-u$ is defined on $\bar{\omega}_{h}$ and satisfies (3), (4) with $\eta_{1}=u-T_{02} u$ and $\eta_{2}=u-$ $T_{20} u$. Now, $\eta_{j}$ is defined on the grid $\omega_{h} \cup \gamma_{h}^{j}$ and equal to zero on $\gamma_{h}^{j}, j=1,2$. Thanks to inequality (5), it suffices to estimate $\left\|\bar{\nabla}_{j} \nabla_{j} \eta_{j}\right\|_{p, h}, j=1,2$. We introduce the squares

$$
\begin{gathered}
E\left(i_{1}, i_{2}\right)=\left\{x=\left(x_{1}, x_{2}\right) \in \mathbf{R}^{2}:\left(i_{j}-1\right) h \leqslant x_{j} \leqslant\left(i_{j}+1\right) h, j=1,2\right\}, \\
E=\left\{t=\left(t_{1}, t_{2}\right) \in \mathbf{R}^{2}:-1 \leqslant t_{j} \leqslant 1, j=1,2\right\},
\end{gathered}
$$

and the affine mapping $x=\left(x_{1}, x_{2}\right) \in E\left(i_{1}, i_{2}\right) \mapsto t=\left(t_{1}, t_{2}\right) \in E$ with $x_{j}=i_{j} h+$ $t_{j} h, j=1,2$. Let us set $\tilde{u}(t)=u(x(t))$. Then

$$
\begin{aligned}
\bar{\nabla}_{1} \nabla_{1} \eta_{1}\left(i_{1} h, i_{2} h\right)= & \frac{u\left(i_{1} h+h, i_{2} h\right)-2 u\left(i_{1} h, i_{2} h\right)+u\left(i_{1} h-h, i_{2} h\right)}{h^{2}} \\
& -\int_{-1}^{1} \theta_{2}(s) \frac{u\left(i_{1} h+h, i_{2} h+s h\right)-2 u\left(i_{1} h, i_{2} h+s h\right)+u\left(i_{1} h-h, i_{2} h+s h\right)}{h^{2}} d s \\
= & \frac{1}{h^{2}}\left\{\tilde{u}(1,0)-2 \tilde{u}(0,0)+\tilde{u}(-1,0)-\int_{-1}^{1} \theta_{2}(s)(\tilde{u}(1, s)-2 \tilde{u}(0, s)+\tilde{u}(-1, s)) d s\right\} .
\end{aligned}
$$


Furthermore, $\bar{\nabla}_{1} \nabla_{1} \eta_{1}\left(i_{1} h, i_{2} h\right)$ is a bounded linear functional on $W^{s, p}(E), s>2 / p$, with a kernel $\supset \mathbf{P}^{3}(E)$. By Lemma 1 ,

$$
\left|\bar{\nabla}_{1} \nabla_{1} \eta_{1}\left(i_{1} h, i_{2} h\right)\right| \leqslant \frac{c}{h^{2}}|\tilde{u}|_{s, p, E}
$$

for $2 / p<s \leqslant 4$. Thus,

$$
\left|\bar{\nabla}_{1} \nabla_{1} \eta_{1}\left(i_{1} h, i_{2} h\right)\right| \leqslant \frac{c}{h^{2}} \cdot h^{s-2 / p}|u|_{s, p, E\left(i_{1}, i_{2}\right)}
$$

for $2 / p<s \leqslant 4$. Finally,

$$
\left\|\bar{\nabla}_{1} \nabla_{1} \eta_{1}\right\|_{p, h} \leqslant c h^{s-2}|u|_{s, p, \Omega}, \quad 2 / p<s \leqslant 4
$$

Likewise,

$$
\left\|\bar{\nabla}_{2} \nabla_{2} \eta_{2}\right\|_{p, h} \leqslant c h^{s-2}|u|_{s, p, \Omega}, \quad 2 / p<s \leqslant 4
$$

and that completes the proof for $k=2$.

(b) Let $k=1$. By (6) it suffices to estimate $\|\left[\nabla_{j} \eta_{j} \|_{p, h}, j=1,2\right.$. In the same manner as in (a) we conclude that

$$
\nabla_{1} \eta_{1}\left(i_{1} h, i_{2} h\right)=\frac{1}{h}\left\{\tilde{u}(1,0)-\tilde{u}(0,0)-\int_{-1}^{1} \theta_{2}(s)(\tilde{u}(1, s)-\tilde{u}(0, s)) d s\right\}
$$

is a bounded linear functional on $W^{s, p}(E), s>2 / p$, with a kernel $\supset \mathbf{P}^{2}(E)$. Therefore,

$$
\mid\left[\nabla_{1} \eta_{1} \|_{p, h} \leqslant c h^{s-1}|u|_{s, p, \Omega}, \quad 2 / p<s \leqslant 3\right.
$$

and, similarly,

$$
\mid\left[\nabla_{2} \eta_{2} \|_{p, h} \leqslant c h^{s-1}|u|_{s, p, \Omega}, \quad 2 / p<s \leqslant 3 .\right.
$$

That completes the proof for $k=1$.

(c) Finally, let $k=0$. Let us estimate $\left\|\eta_{j}\right\|_{p, h}, j=1,2$. Since

$$
\eta_{1}\left(i_{1} h, i_{2} h\right)=\tilde{u}(0,0)-\int_{-1}^{1} \theta_{2}(s) \tilde{u}(0, s) d s
$$

is a bounded linear functional on $W^{s, p}(E), s>2 / p$, with a kernel $\supset \mathbf{P}^{1}(E)$, thanks to Lemma 1 ,

$$
\left\|\eta_{1}\right\|_{p, h} \leqslant c h^{s}|u|_{s, p, \Omega}, \quad 2 / p<s \leqslant 2
$$

and, similarly,

$$
\left\|\eta_{2}\right\|_{p, h} \leqslant c h^{s}|u|_{s, p, \Omega}, \quad 2 / p<s \leqslant 2
$$

By (7) we obtain the desired error estimate.

Lemma 2 enables us to derive scales of error estimates.

THEOREM 2. Let $u$ be the solution of boundary value problem (1), (2) and $v$ the solution of discrete problem (10), (11). If $u \in W^{s, p}(\Omega), 2 / p<s \leqslant 2$ and $0 \leqslant r \leqslant 2$, or $2 / p<s \leqslant 3$ and $1 \leqslant r \leqslant 2,1<p<\infty$, the following error estimate holds

$$
\|u-v\|_{r, p, h} \leqslant C h^{s-r}|u|_{s, p, \Omega},
$$

with a positive constant $C$ independent of $h$. Moreover, if $s \geqslant r$ then finite difference scheme (10), (11) converges in the discrete norm $\|\cdot\|_{r, p . h}$. 
Proof. Let us suppose that $u$ belongs to $W^{s, p}(\Omega), 2 / p<s \leqslant 2,1<p<\infty$ and $0 \leqslant r \leqslant 2$. We apply Lemma 2 with $\alpha=0, \beta=2$ and Theorem 1 to derive the desired estimate.

Likewise, if $u$ belongs to $W^{s, p}(\Omega), 2 / p<s \leqslant 3,1<p<\infty$ and $1 \leqslant r \leqslant 2$, set $\alpha=1$ and $\beta=2$ to conclude the estimate.

Let us turn to finite difference scheme (14), (15).

THEOREM 3. Let $u$ be the solution of boundary value problem (1), (2), $v$ the solution of discrete problem (14), (15), and $k \in\{0,1\}$. If $u \in W^{s, p}(\Omega), 0 \leqslant s<1+1 / p, s \neq$ $1 / p, 1<p<\infty$, then

$$
\left\|T_{11} u-v\right\|_{k, p, h} \leqslant C h^{s-k}|u|_{s, p, \Omega},
$$

with a positive constant $C$ independent of $h$. Moreover, if $s \geqslant k$, then finite difference scheme (14), (15) converges in the discrete norm $\|\cdot\|_{k, p, h}$.

Proof. (a) Let $k=1$. By (12)-(15) it follows that the function $z=v-T_{11} u^{*}$, defined on $\bar{\omega}_{h}$, satisfies (3), (4) with $\eta_{1}=T_{11} u^{*}-T_{31} u^{*}$ and $\eta_{2}=T_{11} u^{*}-T_{13} u^{*}$. The function $\eta_{j}$ is defined on $\omega_{h} \cup \gamma_{h}^{j}$ and equal to zero on $\gamma_{h}^{j}, j=1,2$. We define

$$
\begin{gathered}
E\left(i_{1}, i_{2}\right)=\left\{x=\left(x_{1}, x_{2}\right) \in \mathbf{R}^{2}:\left(i_{j}-\frac{3}{2}\right) h \leqslant x_{j} \leqslant\left(i_{j}+\frac{3}{2}\right) h, j=1,2\right\}, \\
E=\left\{t=\left(t_{1}, t_{2}\right) \in \mathbf{R}^{2}:-\frac{3}{2} \leqslant t_{j} \leqslant \frac{3}{2}, j=1,2\right\}
\end{gathered}
$$

and the affine mapping $x=\left(x_{1}, x_{2}\right) \in E\left(i_{1}, i_{2}\right) \mapsto t=\left(t_{1}, t_{2}\right) \in E$ with $x_{j}=i_{j} h+$ $t_{j} h, j=1,2$. Let $\tilde{u}(t)=u^{*}(x(t))$. Then

$$
\begin{aligned}
\nabla_{1} \eta_{1}\left(i_{1} h, i_{2} h\right)=\frac{1}{h}\{ & \int_{-1 / 2}^{1 / 2} \int_{-1 / 2}^{1 / 2}\left(\tilde{u}\left(s_{1}+1, s_{2}\right)-\tilde{u}\left(s_{1}, s_{2}\right)\right) d s_{1} d s_{2} \\
& \left.\quad-\int_{-1 / 2}^{1 / 2} \int_{-3 / 2}^{3 / 2} \theta_{3}\left(s_{1}\right)\left(\tilde{u}\left(s_{1}+1, s_{2}\right)-\tilde{u}\left(s_{1}, s_{2}\right)\right) d s_{1} d s_{2}\right\}
\end{aligned}
$$

is a bounded linear functional on $W^{s, p}(E), 0 \leqslant s<1+1 / p, s \neq 1 / p$ with a kernel $\supset \mathbf{P}^{2}(E)$. By Lemma 1,

$$
\left|\nabla_{1} \eta_{1}\left(i_{1} h, i_{2} h\right)\right| \leqslant \frac{c}{h}|\tilde{u}|_{s, p, E}, \quad 0 \leqslant s<1+1 / p, s \neq 1 / p .
$$

Thus,

$$
\begin{aligned}
\left|\nabla_{1} \eta_{1}\left(i_{1} h, i_{2} h\right)\right| & \leqslant \frac{c}{h} h^{s-2 / p}\left|u^{*}\right|_{s, p, E\left(i_{1}, i_{2}\right)} \\
& \leqslant \frac{c}{h} h^{s-2 / p}|u|_{s, p, E\left(i_{1}, i_{2}\right) \cap \Omega}, \quad 0 \leqslant s<1+1 / p, s \neq 1 / p,
\end{aligned}
$$

since the extension $u \in \dot{W}^{s, p}(\Omega) \mapsto u^{*} \in W^{s, p}\left(\Omega^{*}\right)$ is continuous for $0 \leqslant s<1+$ $1 / p, s \neq 1 / p, 1<p<\infty$. Finally,

$$
\mid\left[\nabla_{1} \eta_{1} \|_{p, h} \leqslant C h^{s-1}|u|_{s, p, \Omega}, \quad 0 \leqslant s<1+1 / p, s \neq 1 / p .\right.
$$

Similarly,

$$
\mid\left[\nabla_{2} \eta_{2} \|_{p, h} \leqslant C h^{s-1}|u|_{s, p, \Omega}, \quad 0 \leqslant s<1+1 / p, s \neq 1 / p .\right.
$$

By (6) we conclude the desired estimate.

(b) For $k=0$ we apply the same technique as in (a). 
We make use of Theorem 3 and Lemma 2 with $\alpha=0, \beta=1$ to prove the following theorem.

THEOREM 4. Let $u$ be the solution of boundary value problem (1), (2) and $v$ the solution of discrete problem (14), (15). If $u \in W^{s, p}(\Omega), 0 \leqslant s<1+1 / p, s \neq 1 / p$, $1<p<\infty$ and $0 \leqslant r \leqslant 1$, then

$$
\left\|T_{11} u-v\right\|_{r, p, h} \leqslant C h^{s-r}|u|_{s, p, \Omega},
$$

with a positive constant $C$ independent of $h$. Moreover, if $s \geqslant r$, then finite difference scheme (14), (15) converges in the discrete norm $\|\cdot\|_{r, p, h}$.

Remark 7. If $1 / p<s<1+1 / p$, then $f \in W^{s-2, p}(\Omega)$ implies $u \in \dot{W}^{s, p}(\Omega)$ [8]. Furthermore, if $0<s<1 / p$ then $f \in \Xi^{s-2, p}(\Omega)$ implies $u \in W^{s, p}(\Omega)$ [5], [8]. Since $\Delta u^{*}=(\Delta u)^{*}$ for $u \in \dot{W}^{s, p}(\Omega), 0<s<1+1 / p, T_{33} f^{*}$ may be calculated from $f$. Therefore finite difference scheme (14), (15) is applicable.

Institute of Mathematics

University of Belgrade

Studentski trg 16, P.B. 550

11000 Belgrade, Yugoslavia

1. J. H. Bramble \& S. R. Hilbert, "Bounds for a class of linear functionals with applications to Hermite interpolation," Numer. Math., v. 16, 1971, pp. 362-369.

2. F. BREZZI \& D. MARINI, "On the numerical solution of plate bending problems by hybrid methods," RAIRO Anal. Numér., v. 3, 1975, pp. 5-50.

3. T. DuPOnt \& R. Scotr, "Polynomial approximation of functions in Sobolev spaces," Math. Comp., v. 34,1980 , pp. $441-463$.

4. R. D. Lazarov, "On the convergence of finite difference schemes to generalized solutions of the Poisson equation," Differencial'nye Uravnenija, v. 17, 1981, pp. 1285-1294. (Russian)

5. J. L. Lions \& E. Magenes, Non Homogeneous Boundary Value Problems and Applications, Springer-Verlag, Berlin and New York, 1972.

6. J. I. MokIN, "Discrete analogues of imbedding theorems in $W$ classes," Zh. Vychisl. Mat. i Mat. Fiz., v. 11, 1971, pp. 1361-1373. (Russian)

7. L. Schwartz, Theorie des Distributions, Vols. I, II, Hermann, Paris, 1950/1951.

8. H. TRIEBEL, Interpolation Theory, Function Spaces, Differential Operators, VEB Deutscher Verlag der Wissenschaften, Berlin, 1978. 